Pacific

Journal of

Mathematics

GEOMETRIC REALIZATIONS OF FORDY-KULISH NONLINEAR SCHRÖDINGER SYSTEMS

Joel Langer and Ron Perline 


\title{
GEOMETRIC REALIZATIONS OF FORDY-KULISH NONLINEAR SCHRÖDINGER SYSTEMS
}

\author{
Joel Langer And Ron Perline
}

\begin{abstract}
A method of Sym and Pohlmeyer, which produces geometric realizations of many integrable systems, is applied to the Fordy-Kulish generalized non-linear Schrödinger systems associated with Hermitian symmetric spaces. The resulting geometric equations correspond to distinguished arclengthparametrized curves evolving in a Lie algebra, generalizing the localized induction model of vortex filament motion. A natural Frenet theory for such curves is formulated, and the general correspondence between curve evolution and natural curvature evolution is analyzed by means of a geometric recursion operator. An appropriate specialization in the context of the symmetric space $S O(p+2) / S O(p) \times S O(2)$ yields evolution equations for curves in $R^{p+1}$ and $S^{p}$, with natural curvatures satisfying a generalized $m K d V$ system. This example is related to recent constructions of Doliwa and Santini and illuminates certain features of the latter.
\end{abstract}

\section{Introduction.}

Shortly after it was discovered that the Korteweg-deVries equation could be linearized via the spectral transform method [G-G-K-M], Shabat and Zakharov $[\mathbf{S}-\mathbf{Z}]$ showed that the method could also be applied to the (cubic) non-linear Schrödinger equation,

$$
-i \psi_{t}=\psi_{s s}+\frac{1}{2}|\psi|^{2} \psi
$$

Almost concurrently, Hasimoto [Has] discovered the connection between NLS and the localized induction equation (LIE), an idealized model of the evolution of the curved centerline of a thin vortex tube in a three-dimensional ideal fluid. (See [Bat] for a derivation, and [Ric] for the history of this equation, also known as the Betchov-Da Rios equation.) Denoting this evolving centerline by $\gamma(s, t)$ (where $s$ is arclength along the curve and $t$ is time), the curve evolution in this model is described by

$$
\gamma_{t}=\gamma_{s} \times \gamma_{s s}=\kappa B,
$$

where $\kappa(s)$ is the curvature and $B$ the binormal. Recall that along a space curve, the Frenet frame $\{T, N, B\}$ satisfies the equations $T_{s}=\kappa N, \quad N_{s}=$ 
$-\kappa T+\tau B, \quad B_{s}=-\tau N$. The LIE-NLS connection is this: If a curve $\gamma$ with curvature $\kappa$ and torsion $\tau$ evolves according to LIE, then the associated complex curvature function, $\psi=\kappa e^{i \int^{s} \tau(u) d u}$, evolves according to NLS.

In view of the LIE-NLS correspondance, it is not surprising that LIE manifests familiar integrability characteristics, but in geometric form: Soliton solutions, a hierarchy of conserved Hamiltonians in involution, a recursion operator generating the corresponding infinite sequence of commuting Hamiltonian vectorfields - the localized induction hierarchy.

The Hamiltonian nature of LIE itself was introduced by Marsden and Weinstein in $[\mathbf{M}-\mathbf{W}]$; the equation's Poisson geometry was further elucidated in [L-P1]; Yasui and Sasaki developed the structure of LIE in the setting of hereditary operator, Hamiltonian pairs, and master symmetries [S-Y].

Other recent papers have addressed a variety of closely related geometric topics of a more concrete nature, including: Knotted soliton curves of constant torsion $[\mathbf{C}-\mathbf{I}]$; planar, spherical, and constant torsion-preserving curve evolution $[\mathbf{L}]$, [L-P3, L-P4]; integrable variational problems for curves [Lan-S1, Lan-S2, Lan-S3]; pseudospherical surfaces and Weingarten systems [Per1, Per2], evolution of immersed Riemann surfaces in $R^{3}$ preserving the Willmore integral $[\mathbf{G}-\mathbf{L}]$. It is by now clear that the localized induction hierarchy is a rich source of examples and structure in the classical differential geometry of curves and surfaces.

Here we consider natural generalizations of the LIE hierarchy in higher dimensional spaces. Our starting point is the Fordy-Kulish $[\mathbf{F}-\mathbf{K}]$ construction of a generalized nonlinear Schrödinger equation (gNLS) (with spectral problem) associated to a Hermitian symmetric Lie algebra g. We apply a technique due to Sym [Sym] and Pohlmeyer [Pohl], differentiation with respect to the spectral parameter, which produces geometric realizations of many integrable systems. By this route, we arrive at a generalized LIE hierarchy for distinguished arclength-parametrized curves evolving in $\mathbf{g}$, the first three terms of which are:

$$
\begin{gathered}
\gamma_{t}=\gamma_{s}, \\
\gamma_{t}=-\left[\gamma_{s}, \gamma_{s s}\right] \\
\gamma_{t}=-\left(\gamma_{s s s}+\frac{3}{2}\left[\gamma_{s s},\left[\gamma_{s}, \gamma_{s s}\right]\right]\right) .
\end{gathered}
$$

Here, [, ] is the Lie bracket in $\mathbf{g}$, and the subscript $s$ denotes derivative by a curve parameter which is unit speed with respect to the Cartan-Killing form on g. In this setting, a direct generalization of Hasimoto's result is proved (Theorem 3), establishing the correspondence between the above curve evolution equations and evolution of natural curvatures by equations in the gNLS hierarchy; in particular, a curve evolving by the second order flow, gLIE, has curvatures satisfying gNLS. 
Interestingly, as in the three-dimensional case, the odd-order flows are more amenable to geometrically meaningful reductions. In fact, by an ad hoc reduction, in the class of symmetric spaces $S O(p+2) / S O(p) \times S O(2)$, we were able to fully realize our original goal; namely, we obtain geometric evolution equations applicable to arbitrary smooth curves in $E^{n}$ and $S^{n}$. For the third order flow, our equations take the form

$$
\gamma_{t}=-\left(\gamma_{s s s}+\frac{3}{2}\left\|\gamma_{s s}\right\|^{2} \gamma_{s}\right)=-\left(\frac{1}{2} k^{2} T+\sum_{i}\left(u_{i}\right)_{s} U_{i}\right) .
$$

Here, $u_{1}, \ldots, u_{n-1}$ are curvatures belonging to a natural frame $T, U_{1}, \ldots$, $U_{n-1}$. We show (Theorem 5 ) that the corresponding natural curvature vector, $u=\left(u_{1}, \ldots, u_{n-1}\right)$, satisfies the vector modified Korteweg-deVries equation:

$(\mathrm{mKdV})$

$$
u_{t}=-\left(u_{s s s}+\frac{3}{2}|u|^{2} u_{s}\right) .
$$

Note that these simple equations for $\gamma$ and $u$ are given, finally, without reference to a Lie algebra.

We now describe the contents of the paper. Section 2 is a review of the Fordy-Kulish construction of generalized NLS equations. In Section 3, we apply the Sym-Pohlmeyer geometrization procedure in the Fordy-Kulish setting, and develop a natural Frenet theory for the resulting curves. In Section 4, we introduce the geometric recursion operator for the generalized LIE hierarchy, and derive key variation formulas. Section 5 treats the special class of Hermitian symmetric spaces mentioned above, and the reduction yielding curve evolutions in Euclidean spaces and spheres. We note that our constructions in the latter case are related to recent work of Doliwa and Santini $[\mathbf{D}-\mathbf{S}]$; in fact, our investigation developed out of an effort to better understand their equations. Since the completion of our paper, we have learned from Chuu-Lian Terng of her own work (with K. Uhlenbeck) on generalizations of LIE [T-U1, T-U2].

\section{The Fordy-Kulish generalizations of NLS.}

Following a standard framework in the theory of integrable systems, the nonlinear soliton equations arise as compatibility conditions for an overdetermined linear system

$$
\phi_{s}=(\lambda A+Q) \phi, \quad \phi_{t}=V \phi .
$$

This system involves two independent variables, $s$ ("position") and $t$ ("time"), and a scalar $\lambda$, the spectral parameter. The eigenfunction $\phi(s, t ; \lambda)$ has values in a Lie group $\mathbf{G}$, while $U(s, t ; \lambda)=\lambda A+Q(s, t)$ and $V(s, t ; \lambda)$ have values in the Lie algebra $\mathbf{g}$ of $\mathbf{G}$. Here $Q$ is the potential, which is meant to evolve isospectrally, hence the lack of $\lambda$-dependence. For the 
Fordy-Kulish generalized NLS equations, $\mathbf{g}$ is taken to be the compact real form of a complex semi-simple Lie algebra $\mathbf{g}_{C}$; in fact, $\mathbf{g}$ is required to be a Hermitian symmetric Lie algebra, and $A, Q$, are specific to the structure of $\mathbf{g}$. To recall briefly some of the relevant features of this structure, $\mathbf{g}$ has a decomposition as a vector space sum, $\mathbf{g}=\mathbf{k} \oplus \mathbf{m}$, of a compact subalgebra $\mathbf{k}$ and complement $\mathbf{m}$, satisfying the bracket conditions $[\mathbf{k}, \mathbf{k}] \subset \mathbf{k},[\mathbf{m}, \mathbf{m}] \subset \mathbf{k}$, and $[\mathbf{k}, \mathbf{m}] \subset \mathbf{m}$. Also, $\mathbf{k}$ is associated with a special element $A$ in $\mathbf{h}$, a Cartan subalgebra of $\mathbf{g}$; namely, $\mathbf{k}$ is the commutator algebra of $A: \mathbf{k}=\operatorname{kernel}\left(a d_{A}\right)=\{B \in \mathbf{g}:[B, A]=0\}$. Further, $J=a d_{A}$ satisfies $J^{2} \mid \mathbf{m}=-I d$, i.e., $J$ is a complex structure on $\mathbf{m}$. Such an element $A$ is fixed to form (LS) and $Q$ is required to be an $\mathbf{m}$-potential, that is, $Q(s, t) \in \mathbf{m}$ for all $t$. The set of $\mathbf{m}$-potentials $Q(s)$ is clearly a vector space; we will refer to a tangent vectorfield $W$ as a $\mathbf{m}$-field. Some of the above will be explained more explicitly, as required for specializations, below. (Also, see $[\mathbf{F}-\mathbf{K}]$, $[\mathbf{H e l}]$ for more details.)

Cross-differentiating the equations in (LS) gives the zero curvature condition $U_{t}-V_{s}+[U, V]=0$ or

$$
Q_{t}=V_{s}-[\lambda A+Q, V] .
$$

With the aim of finding $V$ in terms of $Q$, such that the compatibility condition (ZC1) is satisfied, a polynomial ansatz is invoked: $V=$ $\sum_{j=0}^{n} P^{(j)}(s, t) \lambda^{n-j}$. (Our indexing convention reverses the order of $[\mathbf{F}-\mathbf{K}]$.) The strategy here is to substitute this expression for $V$ into (ZC1), set the coefficients of powers of $\lambda$ equal to zero, then solve recursively for the $P^{(j)}$ and, finally, obtain a nonlinear PDE for the $\mathbf{m}$-field $Q$ (from the $\lambda^{0}$ term). To carry this out requires the decomposition of $\mathbf{g}$ given above. Namely, each $P^{(j)}$ is decomposed as $P^{(j)}=P_{m}^{(j)}+P_{k}^{(j)}$, with $P_{m}^{(j)} \in \mathbf{m}$ and $P_{k}^{(j)} \in \mathbf{k}$. Then, using the above bracket conditions and $J^{2}=-I d$, one obtains the equations:

$$
\begin{gathered}
P_{m}^{(0)}=0, \\
P_{m}^{(j)}=-J\left(\partial_{s} P_{m}^{(j-1)}-\left[Q, P_{k}^{(j-1)}\right]\right), \quad j=1, \ldots, n, \\
\partial_{s} P_{k}^{(j)}=\left[Q, P_{m}^{(j)}\right], \quad j=0, \ldots, n, \\
Q_{t}=\partial_{s} P_{m}^{(n)}-\left[Q, P_{k}^{(n)}\right] .
\end{gathered}
$$

Note the first and third of these equations imply $P^{(0)}$ is necessarily a constant in $\mathbf{k}$ - we will take the "obvious choice" $P_{k}^{(0)}=P^{(0)}=A$. Also, a choice of "constant of integration" is made, at each stage, as we are apparently required to compute an antiderivative to obtain $P_{k}^{(j)}$. An essential (and remarkable) feature of the recursion scheme is that the antiderivative is explicit, and is polynomial in $Q$ and its derivatives; here, we simply illustrate this point with the important case $n=2$. The required terms are readily generated in the order: $P_{m}^{(1)}=Q, P_{k}^{(1)}=0, P_{m}^{(2)}=-\left[A, Q_{s}\right], P_{k}^{(2)}=$ 
$\frac{1}{2}[Q,[Q, A]]$ (the last antiderivative following from the Jacobi identity). The induced evolution on the $\mathbf{m}$-field $Q$ is then given by

$$
Q_{t}=-J Q_{s s}-\frac{1}{2} a d_{Q}^{3} A,
$$

which we will refer to as the Fordy-Kulish generalized NLS equation. (In $[\mathbf{F}-\mathbf{K}]$, the NLS equations are displayed componentwise, rather than in vector notation.)

The above recursion scheme may be more compactly described by introducing a recursion operator $\tilde{\mathcal{R}}=\tilde{\mathcal{R}}_{Q}$ which takes $\mathbf{m}$-fields $\tilde{X}$ to $\mathbf{m}$-fields:

$$
\tilde{\mathcal{R}} \tilde{X}=-\left(\partial_{s}-a d_{Q} \partial_{s}^{-1} a d_{Q}\right) J \tilde{X} .
$$

(Appropriate specification of the antiderivative $\partial_{s}^{-1}$ depends on the context.) Defining $\tilde{X}^{(j)}=J P_{m}^{(j)}$, we can now write the recursion scheme and nonlinear equations as

$$
\begin{gathered}
\tilde{X}^{(1)}=J Q, \quad \tilde{X}^{(2)}=\tilde{\mathcal{R}} \tilde{X}=Q_{s}, \\
\tilde{X}^{(j+1)}=\tilde{\mathcal{R}} \tilde{X}^{(j)}, \quad j=1,2,3, \ldots, \\
Q_{t}=\tilde{X}^{(j+1)}, \quad j=0,1,2, \ldots
\end{gathered}
$$

The last of these equations defines the $(j+1)$ rst term in the Fordy-Kulish $N L S$ hierarchy, the above NLS equation being the third term.

It will be useful to have a concrete (and particularly simple) example at hand for illustrating the main ideas in the next few sections; thus, we begin our:

\section{Running example.}

For the "classical NLS", we take $\mathbf{g}=s u(2)$. We use the basis $A=\frac{-i}{2} \sigma^{3}$, $B=\frac{-i}{2} \sigma^{1}, C=\frac{-i}{2} \sigma^{2}$, where $\sigma^{1}, \sigma^{2}, \sigma^{3}$ are the Pauli matrices

$$
\sigma^{1}=\left(\begin{array}{ll}
0 & 1 \\
1 & 0
\end{array}\right), \sigma^{2}=\left(\begin{array}{cc}
0 & -i \\
i & 0
\end{array}\right), \sigma^{3}=\left(\begin{array}{cc}
1 & 0 \\
0 & -1
\end{array}\right) .
$$

The bracket relations $[A, B]=C,[B, C]=A,[C, A]=B$, imply that $\mathbf{k}=\operatorname{span}(A)$ and $\mathbf{m}=\operatorname{span}(B, C)$ define a Hermitian symmetric Lie algebra structure on $\mathbf{g}=s u(2)$. Writing $Q=b B+c C$, and plugging into the generalized NLS equation yields:

$$
Q_{t}=-J\left(Q_{s s}+\frac{1}{2}|Q|^{2} Q\right),
$$

where $|Q|^{2}=\left(b^{2}+c^{2}\right)$. In this case, we can identify $\mathbf{m}$ with the complex numbers $(Q$ with $\psi=b+i c)$, and then $J$ coincides with multiplication by $i$. Using this identification (and a time reversal), we obtain exactly (NLS), given in the introduction. 
We conclude this section by recording some useful general identities, to be used in later sections. First of all, we note that the above treatment of the overdetermined linear system LS did not fully reflect the dependence of $\phi$ on the three variables, $s, t$, and $\lambda$. Introducing $W(s, t, \lambda)=\phi_{\lambda} \phi^{-1}$, we write down the augmented linear system

$$
\phi_{s}=U \phi, \quad \phi_{t}=V \phi, \quad \phi_{\lambda}=W \phi,
$$

with corresponding compatibility conditions

$$
U_{t}-V_{s}+[U, V]=V_{\lambda}-W_{t}+[V, W]=W_{s}-U_{\lambda}+[W, U]=0 .
$$

Secondly, the geometric objects related to LS will be expressed in terms of conjugates of $U, V$, and $W$, for which we will use the following notational shorthand: For $B, C \in \mathbf{g}$, we write $\{B\}=\phi^{-1} B \phi$, and $\{B, C\}=\{[B, C]\}$. The following lemma (whose proof involves straighforward differentiation) states analogues of a standard principle of rigid body mechanics (with "time" being $s, t$, or $\lambda)$ : absolute velocity = relative velocity + transferred velocity ([Arn], p. 128).

Proposition 1. For any $\mathbf{g}$ - field $B(s, t, \lambda)$,

$$
\begin{aligned}
& \text { i) }\{B\}_{s}=\left\{B_{s}\right\}+\{B, U\} \text {, } \\
& \text { ii) }\{B\}_{t}=\left\{B_{t}\right\}+\{B, V\} \text {, and } \\
& \text { iii) }\{B\}_{\lambda}=\left\{B_{\lambda}\right\}+\{B, W\} \text {. }
\end{aligned}
$$

Finally, combining Proposition 1 with (ZC2) yields at once six simple identities; three of these we will use, so we collect them in:

Proposition 2. For $U, V$, and $W$ as above, we have

$$
\{V\}_{s}=\left\{U_{t}\right\}, \quad\{W\}_{t}=\left\{V_{\lambda}\right\}, \text { and }\{W\}_{s}=\left\{U_{\lambda}\right\} .
$$

\section{Sym-Pohlmeyer curves.}

Throughout this section, we "freeze time" in the definitions of the previous section. In other words, we consider a time-independent potential $Q(s)$, and suppose $\phi=\phi(s ; \lambda)$ satisfies the linear system $\phi_{s}=U \phi=(\lambda A+Q) \phi$, for each value of the "parameter" $\lambda$. Setting $W(s, \lambda)=\phi_{\lambda} \phi^{-1}$, we consider the g-valued function

$$
\gamma(s, \lambda)=\{W\}=\phi^{-1} \phi_{\lambda} .
$$

By Proposition 2, we have $\gamma_{s}=\{W\}_{s}=\left\{U_{\lambda}\right\}=\{A\}$. If $K$ is the CartanKilling form on $\mathbf{g}, K(B, C)=\operatorname{tr}\left(a d_{B} a d_{C}\right)$, then by $A d$-invariance of $K$, $K\left(\gamma_{s}, \gamma_{s}\right)=K(A, A)=$ constant. In fact, $K(A, A)=\operatorname{tr}\left(\left(a d_{A}\right)^{2}\right)=-d$, where $d=\operatorname{dim}(\mathbf{m})$. Therefore, $\gamma$ will be an arclength-parameterized curve in $\mathbf{g}$ with respect to the rescaled form $\langle\rangle=,-\frac{1}{d} K$. Henceforth, we refer to any curve in the one parameter family $\gamma(s, \lambda)$ as a Sym-Pohlmeyer curve, and denote by $T$ the unit tangent vector $T=\gamma_{s}=\{A\}$. 
To develop a Frenet theory for such curves, we first use Proposition 1 to obtain an expression for the curvature normal $\kappa N$ of a Sym-Pohlmeyer curve,

$$
\kappa N=T_{s}=\{A\}_{s}=\left\{A_{s}\right\}+\{A, \lambda A+Q\}=\{A, Q\}=\{\tilde{Q}\} .
$$

We refer to the $\mathbf{m}$-field $Q$ itself as a curvature coefficients vector. Next, we fix a basis for $\mathrm{g}$ of the form $A_{1}=A, A_{2}, \ldots, A_{c}, B_{1}, \ldots, B_{d}$, where the $A_{i}$ span $\mathbf{k}$ and the $B_{j}$ span $\mathbf{m}$. Since the Killing form $\mathrm{K}$ is definite ( $\mathbf{g}$ is compact), we can further specify the basis to be orthonormal with respect to $\langle$,$\rangle . The curvature normal vector is now expressible as$

$$
\kappa N=\left\{\sum_{j=1}^{d} \kappa_{j} B_{j}\right\}=\sum_{j=1}^{d} \kappa_{j} N_{j}
$$

where $N_{j}=\left\{B_{j}\right\}$.

Next, we write the derivatives of the $N_{j}$,

$$
\left(N_{j}\right)_{s}=\left\{B_{j}\right\}_{s}=\left\{B_{j}, U\right\}=\left\{B_{j}, \lambda A+Q\right\},
$$

as a linear combination of themselves and the vectors $T_{i}=\left\{A_{i}\right\}, i=1, \ldots, c$. Finally, we write the derivatives

$$
\left(T_{i}\right)_{s}=\left\{A_{i}\right\}_{s}=\left\{A_{i}, Q\right\}, i=2, \ldots, c .
$$

At this point, we have a closed system of Frenet equations for the $c+d=$ $\operatorname{dim}(\mathrm{g})$ frame vectors $T_{i}, N_{j}$, involving only the curvature functions $\kappa_{j}$, the spectral parameter $\lambda$, and the structure constants of $\mathbf{g}$.

Running example.

For Sym-Pohlmeyer curves in $\left(s u(2) ;-\frac{1}{2} K\right) \cong\left(R^{3} ;\langle\rangle,\right)$ with curvature vector $Q=b B+c C$, the curvature normal is given by $T_{s}=\kappa N=$ $-c\{B\}+b\{C\}=\kappa_{1} N_{1}+\kappa_{2} N_{2}$, with $\kappa_{1}=-c, \kappa_{2}=b, N_{1}=\{B\}, N_{2}=\{C\}$. Our Frenet system is completed by the two equations $\left(N_{1}\right)_{s}=-\kappa_{1} T-\lambda N_{2}$, and $\left(N_{2}\right)_{s}=-\kappa_{2} T+\lambda N_{1}$. For $\lambda=0$, this is none other than the natural Frenet system for curves in $R^{3}$ (see, e.g., [Bis]). For a general value of the constant $\lambda$, such a system may be thought of as inertial, in that the rigid body defined by $\left\{T, N_{1}, N_{2}\right\}$ (identifying $s$ with time) has constant tangential component of angular velocity. The relationship to the classical Frenet system can be written $\kappa_{1}+i \kappa_{2}=\kappa e^{i \theta}$, and $N_{1}+i N_{2}=(N+i B) e^{i \theta}$, where $\theta=\int^{s} \tau(u)+\lambda d u$; also, $\kappa^{2}=\kappa_{1}^{2}+\kappa_{2}^{2}$ and $\tau=\kappa^{-2}\left(\kappa_{1}\left(\kappa_{2}\right)_{s}-\kappa_{2}\left(\kappa_{1}\right)_{s}\right)-\lambda$. While $\kappa, \tau$ and $\{T, N, B\}$ are uniquely defined along a regular space curve $\gamma$ (with $\kappa \neq 0$ ), the curvatures $\kappa_{1}, \kappa_{2}$ and frame vectors $N_{1}, N_{2}$ are determined (given $\lambda$ ) only up to multiplication by a complex unit, $e^{i \alpha}$ - this freedom corresponds to the choice of antiderivative in the above formulas. Aside from this difference, the natural Frenet theory resulting from these definitions is similar to the classical Fundamental Theorem for space curves. In particular, the set of unit speed curves $\Gamma=\left\{\gamma: R \mapsto R^{3}\right\}$ can be parametrized 
by the following data: initial position $\gamma(0)$, initial frame $T(0), N_{1}(0), N_{2}(0)$, and shape $\kappa_{1}(s), \kappa_{2}(s)$. For a given curve $\gamma$, this data is unique up to choice of arclength parameter $s$, real parameter $\lambda$, and $S^{1}$-parameter $e^{i \theta}$.

How does the Sym-Pohlmeyer construction fit together with the above parametrization of $\Gamma$ ? Since $Q=\kappa_{2} B-\kappa_{1} C$ and $\lambda$ are explicitly part of the construction, it suffices to discuss the initial data $\gamma(0)$, and $T(0)$, $N_{1}(0), N_{2}(0)$. Writing $T=A d_{\phi^{-} 1} A, N_{1}=A d_{\phi^{-1}} B, N_{2}=A d_{\phi^{-} 1} C$, we see that the initial frame is determined by the initial condition on $\phi$, via the adjoint representation of $S U(2)$. In fact, the two-to-one homomorphism $A d: S U(2) \mapsto S O(3)$ implies all initial frames are achieved (twice) as $\phi(0)$ varies over $S U(2)$. Next, allow the initial condition on $\phi$ to depend on $\lambda$, and regard $\phi(0, \lambda)$ as an arbitrary curve in $S U(2)$. Since $\phi^{-1} \phi_{\lambda}$ describes the usual trivialization of the tangent bundle $\operatorname{TSU}(2)$, it follows that $\gamma\left(0, \lambda_{0}\right)=\left.\phi^{-1} \phi_{\lambda}\right|_{\left(0, \lambda_{0}\right)}$ is an arbitrary point in $s u(2) \cong R^{3}$. In conclusion, the Sym-Pohlmeyer curves are precisely the unit speed curves in $R^{3}$, and the correspondence between the Sym-Pohlmeyer construction and $\Gamma$ is fully described.

\section{Remarks.}

1) The above example is prototypical in some, but not all respects. In general, Sym-Pohlmeyer curves constitute a very special subclass of the unit speed curves in $\mathrm{g} \cong R^{c+d}$ - the latter cannot all be described by only $d$ curvatures. In fact, the tangent indicatrix $T(s)$ of a regular curve in $R^{c+d}$ can be any smooth curve in the unit sphere $S^{c+d-1} \subset R^{c+d}$, whereas a SymPohlmeyer curve has tangent of the form $T=\{A\}=A d_{\phi^{-1}} A$. Now the $A d$-orbit of $A$ can be identified with the Hermitian symmetric space $\mathbf{G} / \mathbf{K}$ ( $\mathbf{K}$ having Lie algebra $\mathbf{k}$ ). Thus, the tangent indicatrix of a Sym-Pohlmeyer curve lies in $\mathbf{G} / \mathbf{K} \subset S^{c+d-1} \subset \mathbf{g}$. In special cases, the above procedure may produce a closed system with fewer than $(c+d)$ frame vectors - this will be true of our main construction of Section 5 - and the situation may resemble the example more closely.

2) In the general case, it is reasonable to refer to $k_{1}, \ldots, k_{d}$ as natural curvatures (though this term will have a more special meaning in Section $5)$. Note that the non-uniqueness of natural curvatures is described by the group $\mathbf{K}\left(S U(1)=\left\{e^{i \theta}\right\}\right.$ in the example). Specifically, suppose $\phi_{s}=$ $(\lambda A+Q) \phi$, and consider the Sym-Pohlmeyer curve $\gamma=\phi^{-1} \phi_{\lambda}$. Now let $\varphi=\phi_{0} \phi$, where $\phi_{0} \in \mathbf{K}$ is any constant element. Then $\gamma=\varphi^{-1} \varphi_{\lambda}$, and $\varphi$ satisfies the linear system $\varphi_{s}=\left(\lambda A+A d_{\phi_{0}} Q\right) \varphi$, as is easily checked. So the "gauge transformation" $\phi \mapsto \varphi=\phi_{0} \phi$ leaves the curve $\gamma$ unchanged, while transforming the natural curvatures according to $Q \mapsto A d_{\phi_{0}} Q$. 


\section{The recursion operator and variation formulas.}

Next we "un-freeze" time, and apply the above constructions to time-dependent potentials $Q(s, t)$, obtaining two-parameter families of unit speed curves $\gamma(s, t, \lambda)$. The $t$-derivatives of these will be called Sym-Pohlmeyer (variation) fields. Note that Proposition 2 gives a formula for such vectorfields:

$$
\gamma_{t}=\{W\}_{t}=\left\{V_{\lambda}\right\}
$$

Also, $V$ satisfies the zero curvature equation, $V_{s}=U_{t}+[U, V]=Q_{t}+$ $[\lambda A+Q, V]$. Comparing k-components gives $\left(V_{k}\right)_{s}=\left[Q, V_{m}\right]$, i.e., the kcomponent of $V$ is determined by the $\mathbf{m}$-component of $V$, according to $V_{k}=\partial_{s}^{-1}\left[Q, V_{m}\right]$. Differentiation of this equation by $\lambda$ shows that, similarly, $\left(V_{\lambda}\right)_{k}$ is determined by $\left(V_{\lambda}\right)_{m}:\left(V_{\lambda}\right)_{k}=\partial_{s}^{-1}\left[Q,\left(V_{\lambda}\right)_{m}\right]$. It is convenient to introduce an operator $\mathcal{K}$ which takes $\mathbf{m}$-fields to $\mathbf{k}$-fields:

$$
\mathcal{K}\left(B_{m}\right)=\partial_{s}^{-1}\left[Q, B_{m}\right] .
$$

Thus any Sym-Pohlmeyer field is of the form $Y=\left\{\mathcal{K}\left(B_{m}\right)+B_{m}\right\}$, for some $\mathbf{m}$-field $B_{m}$ (modulo integration constant in $\mathcal{K}$ ). Now the above definitions easily imply the following formula for the arclength derivative of such a Sym-Pohlmeyer field:

$$
Y_{s}=\left\{\left(B_{m}\right)_{s}\right\}+\left\{\mathcal{K}\left(B_{m}\right), Q\right\}+\lambda\left\{B_{m}, A\right\}=\left\{C_{m}\right\},
$$

where $C_{m}$ is an $\mathbf{m}$-field.

Remark. The result just obtained has the following (partial) interpretation in the context of curve geometry (in a Riemannian manifold). Suppose $\gamma(s, t)$ is any one-parameter family of arclength parametrized curves, and let $X$ be the vectorfield $X=\partial_{t} \gamma$. Then $X_{s}$ (the covariant derivative of $X$ with respect to the unit tangent $T$ ) has no tangential component; in fact, the condition for a vectorfield $X$ to be locally arclength preserving $([\mathbf{L}-\mathbf{P} 2])$ is $\left\langle X_{s}, T\right\rangle=0$. Of course, a Sym-Pohlmeyer field satisfies this condition: $\left\langle T, Y_{s}\right\rangle=\left\langle\{A\},\left\{C_{m}\right\}\right\rangle=\left\langle A, C_{m}\right\rangle=0$, since $\mathbf{k}$ and $\mathbf{m}$ are orthogonal with respect to the Killing form. In the special case $c=1$, the Sym-Pohlmeyer fields are exactly the locally arclength preserving vectorfields, while for $c>1$, the Sym-Pohlmeyer vectorfields form a strictly smaller class of vectorfields.

For a Sym-Pohlmeyer curve $\gamma$ in a Hermitian symmetric Lie algebra g, we now define three operators on vectorfields $Y=\{B\}$ along $\gamma$.

(i) renormalization operator:

$$
\mathcal{P}(\{B\})=\left\{\mathcal{K}\left(B_{m}\right)+B_{m}\right\}=\left\{\partial_{s}^{-1}\left[Q, B_{m}\right]+B_{m}\right\} ;
$$

(ii) geometric recursion operator:

$$
\mathcal{R} Y=-\mathcal{P}\left(\left[T, \partial_{s} Y\right]\right)
$$


(iii) intertwining operator:

$$
\mathcal{Z}(Y)=a d_{A}\left(A d_{\phi} Y\right)
$$

The next lemma explains the nomenclature for $\mathcal{Z}$ :

Proposition 3. For $Y$ a Sym-Pohlmeyer field,

$$
\mathcal{Z R} Y=(\tilde{\mathcal{R}}-\lambda) \mathcal{Z} Y \text {. }
$$

Proof. Using the above computation of $Y_{s}$, we have

$$
\begin{aligned}
\mathcal{Z R} Y & =-\mathcal{Z P}\left(\left[T, Y_{s}\right]\right) \\
& =-\mathcal{Z}\left(\left\{A,\left(B_{m}\right)_{s}+\left[\mathcal{K}\left(B_{m}\right), Q\right]+\lambda\left[B_{m}, A\right]\right\}\right) \\
& =-\mathcal{Z}\left(\left\{J\left(\left(B_{m}\right)_{s}+\left[\mathcal{K}\left(B_{m}\right), Q\right]\right)+\lambda B_{m}\right\}\right) \\
& =-J\left(J\left(\left(B_{m}\right)_{s}-a d_{Q} \partial_{s}^{-1} a d_{Q} B_{m}\right)+\lambda B_{m}\right) \\
& =\left(\partial_{s}-a d_{Q} \partial_{s}^{-1} a d_{Q}\right) B_{m}-\lambda J B_{m} \\
& =-\left(\partial_{s}-a d_{Q} \partial_{s}^{-1} a d_{Q}\right) J^{2} B_{m}-J \lambda B_{m} \\
& =(\tilde{\mathcal{R}}-\lambda) J B_{m}=(\tilde{\mathcal{R}}-\lambda) \mathcal{Z} Y .
\end{aligned}
$$

Next, we consider a Sym-Pohlmeyer variation, $\gamma(s, t, \lambda)=\phi^{-1} \phi_{\lambda}=\{W\}$, and the corresponding Sym-Pohlmeyer field (infinitesimal variation) $X=$ $\gamma_{t}=\left\{V_{\lambda}\right\}$.

Proposition 4. $\mathcal{R} X=\{V+\tilde{A}\}$, for some constant $\tilde{A} \in \mathbf{k}$.

Proof.

$$
\begin{aligned}
\mathcal{R} X & =-\mathcal{P}\left(\left[T, \frac{\partial}{\partial s} \frac{\partial}{\partial t} \gamma\right]\right)=-\mathcal{P}\left(\left[T, T_{t}\right]\right) \\
& =-\mathcal{P}\left(\left[T,\{A\}_{t}\right]\right)=-\mathcal{P}([T,\{A, V\}]) \\
& =-\mathcal{P}(\{A,[A, V]\})=-\mathcal{P}\left(\left\{J^{2} V\right\}\right) \\
& =\mathcal{P}\left(\left\{V_{m}\right\}\right)=\left\{\mathcal{K}\left(V_{m}\right)+V_{m}\right\}=\{V+\tilde{A}\} .
\end{aligned}
$$

This last step uses $\mathcal{K}\left(V_{m}\right)=V_{k}+\tilde{A}$, as observed above, with the arbitrary "integration constant" $\tilde{A} \in \mathbf{k}$ explicitly displayed here.

Theorem 1. Variation of curvatures formula:

The time variation of the "curvature coefficients vector" $Q$ induced by a Sym-Pohlmeyer field $X=\gamma_{t}=\left\{V_{\lambda}\right\}$ is given by

$$
Q_{t}=\mathcal{Z R}^{2} X+[Q, \tilde{A}] .
$$

In the "gauge term", $[Q, \tilde{A}], \tilde{A} \in \mathbf{k}$ is a constant.

Proof. Combining Propositions 2 and 4, we have

$$
\begin{aligned}
\mathcal{Z R}^{2}\left(\gamma_{t}\right) & =\mathcal{Z} \mathcal{R}(\{V+\tilde{A}\})=-\mathcal{Z P}\left(\left[T,\{V+\tilde{A}\}_{s}\right]\right) \\
& =-\mathcal{Z P}\left(\left[T,\left\{Q_{t}\right\}+\{\tilde{A}, Q\}\right]\right)=-\mathcal{Z P}\left(\left\{A, Q_{t}+[\tilde{A}, Q]\right\}\right)
\end{aligned}
$$




$$
=-\left(a d_{A}\right)^{2}\left(Q_{t}+[\tilde{A}, Q]\right)=Q_{t}+[\tilde{A}, Q] .
$$

The term $[Q, \tilde{A}]$ can be interpreted as follows. As explained in Remark 2 of the previous section, the non-uniqueness of natural curvatures for a given curve corresponds to the set of transformations $Q \mapsto A d_{\phi_{0}} Q$, where $\phi_{0} \in \mathbf{K}$ is a constant. For a curve evolving in time $t, \phi_{0}$ should be treated as a function of $t$ as well (with initial value $\left.\phi_{0}\right|_{t=0}=I d$ ). Differentiation of $\phi_{0}$ with respect to $t$ results in the term $\left[\left.\left(\phi_{0}\right)_{t}\right|_{t=0}, Q\right]$ in the infinitesimal variation of $A d_{\phi_{0}} Q$.

The appearance of the square of the recursion operator in this formula suggests that between the curve $\gamma$ and curvature coefficients vector $Q$, there is an intermediate object whose variation ought to be considered in this context. The appropriate intermediate object is a Sym-Pohlmeyer frame $T_{i}=\left\{K_{i}\right\}, i=1, \ldots, c, N_{j}=\left\{M_{j}\right\}, j=1, \ldots, d$, as considered above.

Theorem 2. Variation of Frames formula:

If $B \in \mathbf{g}$ is constant, then the time variation of $F=\{B\}$ induced by a Sym-Pohlmeyer field $X=\gamma_{t}$ is given by

$$
F_{t}=[F, \mathcal{R} X]+\{\tilde{A}, B\},
$$

for some constant $\tilde{A} \in \mathbf{k}$; i.e., $\mathcal{R} X$ is essentially the "Darboux vector" for any Sym-Pohlmeyer frame along $\gamma$.

Proof. Using Propositions 1 and 4, we compute

$$
F_{t}=\{B, V\}=[\{B\},\{V\}]=[F, \mathcal{R} X]+\{\tilde{A}, B\} .
$$

We are now in a position to geometrize the Fordy-Kulish NLS hierarchy, the first few terms of which we list here for convenience:

$$
\begin{aligned}
& \tilde{X}^{(1)}=J Q, \\
& \tilde{X}^{(2)}=Q_{s}, \\
& \tilde{X}^{(3)}=-J Q_{s s}-\frac{1}{2} a d_{Q}^{3} A, \\
& \tilde{X}^{(n+1)}=\tilde{\mathcal{R}} \tilde{X}^{(n)} .
\end{aligned}
$$

For a Sym-Pohlmeyer curve $\gamma$ with curvature vector $Q$ and with $\lambda=0$, let vector fields $X^{n}$ be defined along $\gamma$ according to:

$$
\begin{aligned}
& X^{(0)}=\{A\}=T \\
& X^{(1)}=\{Q\}=-\left[\gamma_{s}, \gamma_{s s}\right], \\
& X^{(2)}=-\left\{-\frac{1}{2}[Q,[Q, A]]+\left[A, Q_{s}\right]\right\}=-\left(\gamma_{s s s}+\frac{3}{2}\left[\gamma_{s s},\left[\gamma_{s}, \gamma_{s s}\right]\right]\right), \\
& X^{(n+1)}=\mathcal{R} X^{(n)} .
\end{aligned}
$$


Now consider the curve evolution equation $\gamma_{t}=X^{(n)}$. By Theorem 1 and Proposition 3 , we can write the corresponding curvature evolution as

$$
\begin{aligned}
Q_{t} & =\mathcal{Z} \mathcal{R}^{2} X^{(n)}+[Q, \tilde{A}]=\mathcal{Z} \mathcal{R}^{(n+1)} X^{(1)}+[Q, \tilde{A}] \\
& =\tilde{\mathcal{R}}^{(n+1)} \mathcal{Z} X^{(1)}+[Q, \tilde{A}]=\tilde{\mathcal{R}}^{(n+1)} \tilde{X}^{(1)}+[Q, \tilde{A}] \\
& =\tilde{X}^{(n+2)}+[Q, \tilde{A}] .
\end{aligned}
$$

We summarize this result (suppressing the gauge term, $[Q, \tilde{A}])$ as:

Theorem 3. Evolution of a Sym-Pohlmeyer curve (with $\lambda=0)$ by $\gamma_{t}=$ $X^{(n)}$ corresponds to curvature evolution by $Q_{t}=\tilde{X}^{(n+2)}$. In particular, the generalized LIE,

$$
\gamma_{t}=-\left[\gamma_{s}, \gamma_{s s}\right]
$$

corresponds to the curvature evolution by $g N L S, Q_{t}=-J Q_{s s}-\frac{1}{2} a d_{Q}^{3} A$ (the analogue of Hasimoto's result).

\section{Running example.}

The geometric recursion operator for curves in $R^{3}$ can be written: $\mathcal{R} X=$ $-\mathcal{P}\left(T \times \frac{\partial}{\partial s} X\right)$. Here, $\times$ is the cross product in $R^{3}$, and the reparameterization operator $\mathcal{P}$ turns an arbitrary vectorfield along $\gamma, Y=f T+g U+h V$, into a locally arclength preserving vectorfield, $\mathcal{P} Y=\int^{s}\left(\kappa_{1} g+\kappa_{2} h\right) d s T+$ $g U+h V$, by changing only the tangential component. Using the identification of $\mathbf{m}$ with the complex plane, the operator $\mathcal{Z}$ may be regarded as a simple isomorphism between normal vectorfields $Y=g N_{1}+h N_{2}$ and complex functions $\mathcal{Z}(Y)=i(g+i h)$. On the other hand, $Q$ has already been identified with the complex function $\psi=b+i c=\kappa_{2}-i \kappa_{1}$. Using these definitions, the infinitesimal variation of $\psi$ induced by the vectorfield $X=\gamma_{t}$ may be written as: $\frac{\partial}{\partial t} \psi=\mathcal{Z R}^{2} X+i r \psi, r$ a real constant. This differential formula for the Hasimoto transformation easily implies, e.g., that if $\gamma(s, t)$ evolves by LIE, $\frac{\partial}{\partial t} \gamma=\kappa B=-\kappa_{2} N_{1}+\kappa_{1} N_{2}$, then $\psi($ with $\lambda=0)$ evolves according to NLS. [There is a minor difference between the formulas discussed here and those of $[\mathbf{L}-\mathbf{P} 1, \mathbf{L}-\mathbf{P} 2, \mathbf{L}-\mathbf{P} 3]$. In those references, $\psi$ was the "complex curvature function" $\kappa_{1}+i \kappa_{2}=i(b+i c)$ mentioned in the introduction. The differential formula was written in terms of this $\psi$ (with a minor difference in the definition of $\mathcal{Z}$ ); if $\frac{\partial}{\partial t} \gamma=\kappa B$, then NLS is satisfied by the latter $\psi$ as well (NLS being $i$-equivariant).]

\section{Evolution of curves in $R^{p+1}$ and $S^{p}$.}

We have seen that the geometric realization of the Fordy-Kulish NLS hierarchy is a sequence of evolution equations on the space of Sym-Pohlmeyer curves in a (real compact) Lie algebra g. The Sym-Pohlmeyer curves have curvature vectors $Q$ which are $\mathbf{m}$-valued. As stated in Section 3, SymPohlmeyer curves in general form a proper subset of the set of all arc-length 
parameterized curves in the Lie algebra $\mathbf{g}$. In this section we describe a specific instance of our constructions which allows for a more complete analysis and full geometric interpretation. We will consider the Lie algebra $\mathbf{g}=s o(p+2)$ with subalgebra $\mathbf{k}=s o(p) \oplus s o(2)$ corresponding to the Hermitian symmetric space $B D I$. After describing the relevant structure and commutation relations in appropriate detail, we give an explicit formula for the the operator $\tilde{\mathcal{R}}^{2}$ restricted to a distinguished subspace of $\mathbf{m}$-fields. We then show that the Sym-Polhmeyer curves associated with appropriately restricted curvature functions can be naturally considered as corresponding to all curves in the Euclidean space $R^{p+1}$, and the geometric realizations of the terms in an associated $m K d V$ hierarchy appear as quite natural evolution equations on curves. We explicitly compute the first non-trivial term, and show that it induces curvature evolution corresponding to a particularly simple coupled $\mathrm{mKdV}$ system. This $\mathrm{mKdV}$ system is a rather special reduction of a system which fits into the general framework of $[\mathbf{F}-\mathbf{K}],[\mathbf{A}-\mathbf{F}]$ (though $B D I$ is an exceptional case in the framework of $[\mathbf{A}-\mathbf{F}]$ ).

We consider $s o(p+2)$ lying in $g l(p+2, R)$. We have $g l(p+2, R)$ commutation relations $\left[e_{j, k}, e_{l, m}\right]=\delta_{k, l} e_{j, m}-\delta_{m, j} e_{l, k}, 1 \leq j, k, l, m \leq p+2$, where $e_{j, k}$ is the matrix with 1 in the $j$ th row, $k t h$ column, zero otherwise. Setting $f_{i, j}=e_{i, j}-e_{j, i}$, we can express $s o(p+2)$ commutation relations in the form

$$
\left[f_{j, k}, f_{l, m}\right]=\delta_{j, m} f_{k, l}+\delta_{k, l} f_{j, m}-\delta_{j, l} f_{k, m}-\delta_{k, m} f_{j, l} .
$$

As it turns out, in addition to the natural notation for the $s o(p+2)$ basis, $\left\{f_{i, j}\right\}, 1 \leq i<j \leq p+2$, it will be convenient to have a notation adapted to a particular decomposition of $s o(p+2)$; thus we define

$$
\begin{aligned}
A & =f_{1,2}, \\
X_{j} & =f_{1, j+2}, j=1, \ldots, p, \\
Y_{k} & =f_{k+2,2}, k=1, \ldots, p, \\
K_{m, n} & =f_{m+2, n+2}, m, n=1, \ldots, p .
\end{aligned}
$$

The $s o(p+2)$ commutation relations now take the form:

$$
\begin{aligned}
{\left[A, X_{j}\right] } & =Y_{j}, \quad\left[A, Y_{j}\right]=-X_{j}, \quad\left[A, K_{m, n}\right]=0, \\
{\left[X_{j}, Y_{k}\right] } & =\delta_{j, k} A, \\
{\left[X_{j}, X_{k}\right] } & =\left[Y_{j}, Y_{k}\right]=-K_{j, k}, \\
{\left[X_{j}, K_{m, n}\right] } & =\delta_{j, m} X_{n}-\delta_{j, n} X_{m}, \\
{\left[Y_{j}, K_{m, n}\right] } & =\delta_{j, m} Y_{n}-\delta_{j, n} Y_{m}, \\
{\left[K_{j, k}, K_{l, m}\right] } & =\delta_{j, m} K_{k, l}+\delta_{k, l} K_{j, m}-\delta_{j, l} K_{k, m}-\delta_{k, m} K_{j, l} .
\end{aligned}
$$

Now consider the following subspaces of $\mathbf{g}=s o(p+2)$ :

$$
\begin{gathered}
\mathbf{k}=\operatorname{span}\{A\} \oplus \operatorname{span}\left\{K_{m, n}\right\}, \\
\mathbf{m}_{x}=\operatorname{span}\left\{X_{j}\right\}, \quad \mathbf{m}_{y}=\operatorname{span}\left\{Y_{k}\right\},
\end{gathered}
$$


and

$$
\mathbf{m}=\mathbf{m}_{x} \oplus \mathbf{m}_{y} .
$$

Part of the structure implicit in these definitions is summarized in

\section{Proposition 5.}

i) $\mathbf{g}=\mathbf{k} \oplus \mathbf{m}$, and $\mathbf{k}=s o(2) \oplus s o(p)$;

ii) $\mathbf{k}$ is the commutator subalgebra of $A$ in $\mathbf{g}$;

iii) $J=a d_{A} \mid \mathbf{m}$ satisfies $J^{2}=-I$.

In particular, $\mathrm{g}$ admits a Hermitian symmetric Lie algebra structure (as defined in Section 2).

Remark. The proposition does not fully capture all the relevant structure of $\mathbf{g}$ for the geometric considerations to follow. In this connection it should be noted that the same Hermitian symmetric Lie algebra $\mathbf{g}=\mathbf{k} \oplus \mathbf{m}$ arises as a byproduct of the standard construction of $s o(p+2)$ as compact real form of $s o(p+2, C)$. However, a different $X, Y$-decomposition of $\mathbf{m}$ appears, which lacks the required properties; specifically, the $X, Y$-bracket relations are not as simple as above.

Next, recall the recursion operator $\tilde{\mathcal{R}}=\tilde{\mathcal{R}}_{Q}$ (first introduced in Section 2) which takes an $\mathbf{m}$-field $\tilde{X}$ to an $\mathbf{m}$-field $\tilde{\mathcal{R}} \tilde{X}$ (and which depends on the m-potential $Q$ ). Henceforth, we adopt the following:

Specialization. $Q$ is an $\mathbf{m}_{x}$-valued potential (more briefly, an $\mathbf{m}_{x}$-potential) and $\tilde{X}$ is an $\mathbf{m}_{x}$-field.

The fact that this specialization is preserved by $\tilde{\mathcal{R}}^{2}$ is an immediate consequence of the following:

Proposition 6. Let $Q=\sum_{k} u_{k}(s) X_{k}$, and $\tilde{X}=\sum_{m} x_{m}(s) X_{m}$. Then

i) $\tilde{\mathcal{R}} \tilde{X}=-\sum_{k}\left(\partial_{s} x_{k}+u_{k} \sum_{l} \partial_{s}^{-1}\left(u_{l} x_{l}\right)\right) Y_{k}$;

ii) $\tilde{\mathcal{R}}^{2} \tilde{X}=-\sum_{k}\left(\partial_{s}^{2} x_{k}+\sum_{l}\left(\partial_{s}\left(u_{k} \partial_{s}^{-1}\left(u_{l} x_{l}\right)\right)\right.\right.$

$$
\left.\left.+u_{l} \partial_{s}^{-1}\left(u_{l} \partial_{s} x_{k}-u_{k} \partial_{s} x_{l}\right)\right)\right) X_{k}
$$

Proof. The proof is by straightforward computation; however, we include it, since (i) depends on the nice bracket formula $\left[X_{j}, Y_{k}\right]=\delta_{j, k} A$, and (ii) involves a noteworthy cancellation. Consider $\tilde{\mathcal{R}} \tilde{X}=\left(-\partial_{s} J+a d_{Q} \partial_{s}^{-1} a d_{Q} J\right) \tilde{X}$. The first term, $-\partial_{s} J \tilde{X}$, can immediately be written as $-\sum_{k} \partial_{s} x_{k} Y_{k}$. The second term can be rewritten as

$$
\begin{aligned}
a d_{Q} \partial_{s}^{-1} a d_{Q} J \tilde{X} & =\sum_{k, l, m} u_{k} \partial_{s}^{-1}\left(u_{l} x_{m}\right)\left[X_{k},\left[X_{l}, Y_{m}\right]\right] \\
& =\sum_{k, l, m} u_{k} \partial_{s}^{-1}\left(u_{l} x_{m}\right)\left[X_{k}, \delta_{l, m} A\right]
\end{aligned}
$$




$$
\begin{aligned}
& =-\sum_{k, l, m} u_{k} \partial_{s}^{-1}\left(u_{l} x_{m}\right) \delta_{l, m} Y_{k} \\
& =-\sum_{k} u_{k} \sum_{l} \partial_{s}^{-1}\left(u_{l} x_{l}\right) Y_{k} .
\end{aligned}
$$

Summing these two terms gives the desired formula (i).

To prove (ii), write $\tilde{\mathcal{R}} \tilde{\mathcal{R}} \tilde{X}=\left(-\partial_{s} J+a d_{Q} \partial_{s}^{-1} a d_{Q} J\right) \tilde{\mathcal{R}} \tilde{X}$, and note that the first term is $-\partial_{s} J \tilde{\mathcal{R}} \tilde{X}=-\partial_{s}\left(\sum_{k}\left(\partial_{s} x_{k}+u_{k} \sum_{l} \partial_{s}^{-1}\left(u_{l} x_{l}\right)\right) X_{k}\right)$; this accounts for the first two terms of (ii). Next, using $\left[X_{i},\left[X_{j}, X_{k}\right]\right]=\left[X_{i},-K_{j, k}\right]=$ $\delta_{i, k} X_{j}-\delta_{i, j} X_{k}$, one computes

$$
\begin{aligned}
a d_{Q} \partial_{s}^{-1} a d_{Q} J \tilde{\mathcal{R}} \tilde{X} & =\sum_{i, j, k} u_{i} \partial_{s}^{-1}\left(u_{j}\left(\partial_{s} x_{k}+u_{k} \sum_{r} \partial_{s}^{-1}\left(u_{r} x_{r}\right)\right)\right)\left(\delta_{i, k} X_{j}-\delta_{i, j} X_{k}\right) \\
= & \sum_{l, j} u_{l} \partial_{s}^{-1}\left(u_{j}\left(\partial_{s} x_{l}+u_{l} \sum_{r} \partial_{s}^{-1}\left(u_{r} x_{r}\right)\right)\right) X_{j} \\
& -\sum_{l, k} u_{l} \partial_{s}^{-1}\left(u_{l}\left(\partial_{s} x_{k}+u_{k} \sum_{r} \partial_{s}^{-1}\left(u_{r} x_{r}\right)\right)\right) X_{k} \\
= & \sum_{l, k} u_{l} \partial_{s}^{-1}\left(u_{k}\left(\partial_{s} x_{l}+u_{l} \sum_{r} \partial_{s}^{-1}\left(u_{r} x_{r}\right)\right)\right. \\
& \left.-u_{l}\left(\partial_{s} x_{k}+u_{k} \sum_{r} \partial_{s}^{-1}\left(u_{r} x_{r}\right)\right)\right) X_{k} \\
= & -\sum_{l, k} u_{l} \partial_{s}^{-1}\left(u_{l} \partial_{s} x_{k}-u_{k} \partial_{s} x_{l}\right) X_{k},
\end{aligned}
$$

which is the last term of formula (ii).

We return now to the Fordy-Kulish NLS hierarchy. The second evolution equation, $Q_{t}=\tilde{X}^{(2)}=Q_{s}$ evidently preserves the space of $\mathbf{m}_{x}$-potentials. In fact, writing $Q=\sum_{i} u_{i}(s) X_{i}$, the resulting evolution for the components $u_{i}$ is given by $\left(u_{i}\right)_{t}=\left(u_{i}\right)_{s}$.

From the proposition, it now follows that the evolution $Q_{t}=\tilde{X}^{(4)}=$ $\tilde{\mathcal{R}}^{2} \tilde{X}^{(2)}$ also preserves the space of $\mathbf{m}_{x}$-potentials, and the same is true for all of the even evolution equations $Q_{t}=\tilde{X}^{(2 n)}$. In particular, we can apply formula (ii) to the $\mathbf{m}_{x}$-field $\tilde{X}^{(2)}=\sum_{i}\left(u_{i}\right)_{s} X_{i}$, obtaining

$$
\tilde{X}^{(4)}=-\sum_{k}\left(\partial_{s}^{3} u_{k}+\sum_{l}\left(\partial_{s}\left(u_{k} \partial_{s}^{-1}\left(u_{l} \partial_{s} u_{l}\right)\right)\right.\right.
$$




$$
\left.\left.+u_{l} \partial_{s}^{-1}\left(u_{l} \partial_{s}^{2} u_{k}-u_{k} \partial_{s}^{2} u_{l}\right)\right)\right) X_{k}
$$

Substituting $\frac{1}{2} u_{l}^{2}$ for $\partial_{s}^{-1}\left(u_{l} \partial_{s} u_{l}\right)$, and $u_{l} \partial_{s} u_{k}-u_{k} \partial_{s} u_{l}$ for $\partial_{s}^{-1}\left(u_{l} \partial_{s}^{2} u_{k}-\right.$ $\left.u_{k} \partial_{s}^{2} u_{l}\right)$, we obtain a simpler expression for $\tilde{X}^{(4)}$ :

$$
\tilde{X}^{(4)}=-\sum_{k}\left(\partial_{s}^{3} u_{k}+\frac{3}{2} \sum_{l} u_{l}^{2} \partial_{s} u_{k}\right) X_{k} .
$$

It follows that the evolution equation $Q_{t}=\tilde{X}^{(4)}$ in terms of the components $u_{i}$ is a modified Korteweg-deVries system:

$(\mathrm{mKdVS})$

$$
\left(u_{i}\right)_{t}=-\left(\partial_{s}^{3} u_{i}+\frac{3}{2} \sum_{l} u_{l}^{2} \partial_{s} u_{i}\right), i=1, \ldots, p .
$$

Remark. So far, we have seen that the space of $\mathbf{m}_{x}$-fields (along $\mathbf{m}_{x^{-}}$ potentials) is preserved by the operator $\tilde{\mathcal{R}}^{2}$; as a consequence, the even terms in the NLS hierarchy induce evolution equations on the space of $\mathbf{m}_{x^{-}}$ potentials. It turns out that a corresponding result holds in the general setting of Hermitian symmetric Lie algebras, with the X, Y-decomposition of $\mathbf{m}$ mentioned in the previous remark. For reasons already given, one cannot generally expect such simple formulas and equations corresponding to those just presented. But from our point of view, the most important difference with the present case shows up in the construction of Sym-Pohlmeyer curves from curvature data $Q$. Note the key role of the $X, Y$-bracket relations in the following

Proposition 7. Consider an $\mathbf{m}_{x}$-potential $Q=\sum_{i} u_{i} X_{i}$ with associated Sym-Pohlmeyer curve $\gamma$ (with $\lambda=0)$. The unit tangent vectorfield $T=$ $\{A\}$ and vectorfields $U_{i}=\left\{Y_{i}\right\}, i=1, \ldots, p$, defined along $\gamma$, satisfy the following closed, linear system:

$$
\begin{aligned}
T_{s} & =\sum_{i} u_{i} U_{i}, \\
\left(U_{i}\right)_{s} & =-u_{i} T .
\end{aligned}
$$

Proof. We have

$$
\begin{aligned}
T_{s}=\{A\}_{s} & =\left\{A_{s}\right\}+\{[A, Q]\} \\
& =\left\{\left[A, \sum_{i} u_{i} X_{i}\right]\right\} \\
& =\sum_{i} u_{i}\left\{Y_{i}\right\}=\sum_{i} u_{i} U_{i} ; \\
\left(U_{i}\right)_{s}=\left\{Y_{i}\right\}_{s} & =\left\{\left(Y_{i}\right)_{s}\right\}+\left\{\left[Y_{i}, Q\right]\right\}
\end{aligned}
$$




$$
\begin{aligned}
& =\left\{Y_{i}, \sum_{j} u_{j} X_{j}\right\} \\
& =\sum_{j} u_{j}\left\{\left[Y_{i}, X_{j}\right]\right\} \\
& =-\sum_{j} u_{j} \delta_{i, j}\{A\}=-u_{i}\{A\}=-u_{i} T .
\end{aligned}
$$

Let $\Psi$ be a $(p+1) \times(p+1)$ matrix which is the fundamental solution to the matrix differential equation

$$
\begin{aligned}
\Psi_{s} & =\left(\begin{array}{cccc}
0 & u_{1} & \cdots & u_{p} \\
-u_{1} & 0 & \cdots & 0 \\
\vdots & \vdots & & \vdots \\
-u_{p} & 0 & \cdots & 0
\end{array}\right) \Psi \\
\Psi\left(s_{0}\right) & =I_{p+1}=(p+1) \times(p+1) \text { identity matrix. }
\end{aligned}
$$

Then by the fundamental theorem of differential equations, we can express the moving frame $T(s), U_{i}(s)$ as

$$
\left(\begin{array}{c}
T(s) \\
U_{1}(s) \\
\vdots \\
U_{p}(s)
\end{array}\right)=\Psi\left(\begin{array}{c}
T\left(s_{0}\right) \\
U_{1}\left(s_{0}\right) \\
\vdots \\
U_{p}\left(s_{0}\right)
\end{array}\right) .
$$

It follows that the Sym-Pohlmeyer curve $\gamma \subset \mathbf{g}$ actually lies in the affine space $\gamma\left(s_{0}\right)+R^{p+1}$, where $R^{p+1}$ is here identified as the span of $T\left(s_{0}\right), U_{i}\left(s_{0}\right), i=1, \ldots, p$. Moreover, the equations given in the proposition are the natural Frenet equations (see Running example in Section 3) for a curve in $R^{p+1}$ with curvatures $u_{i}(s), i=1, \ldots, p$ and natural frame $T, U_{i}, i=1, \ldots, p$. Thus, we conclude that the Sym-Pohlmeyer curve $\gamma$ may be regarded as a general space curve in $R^{p+1}$.

Since the natural Frenet equations are not so well-known, we take a moment to indicate some of their geometric significance. To begin with, the conclusion just reached depends on an analogue of the classical Fundamental Theorem of Curve Theory (for curves in n-dimensional Euclidean space). In particular, every smooth curve $\gamma$ in $R^{p+1}$ satisfies the above system for some choice of curvature functions $u_{i}(s), i=1, \ldots, p$, and is uniquely determined, up to congruence, by these functions. (The converse statement differs a bit from that of the classical theorem, in that the natural curvatures $u_{i}$ are uniquely determined by a curve $\gamma$ only after the frame $T\left(s_{0}\right), U_{i}\left(s_{0}\right)$ has been specified at some initial point $\gamma\left(s_{0}\right)$.) Note that $T_{s}=\kappa N=\sum_{i} u_{i} U_{i}$, 
implies $\kappa^{2}=\sum_{i}\left(u_{i}\right)^{2}$, so one can recover the standard (first) curvature from natural curvatures.

What's more important, the curvatures $u_{i}$ measure the sphericity of a curve in $R^{p+1}$. In particular, suppose that, for some $j, u_{j}=c_{j}=$ constant $\neq$ 0 . Then $\left(\gamma(s)+\left(1 / c_{j}\right) U_{j}\right)_{s}=T+\left(1 / c_{j}\right) c_{j}(-T)=0$, so $\gamma$ lies on a $p$ dimensional sphere of radius $1 / c_{j}$. Further, $U_{j}$ is the inward pointing unit normal to the sphere along $\gamma$, and the remaining frame vectors determine a natural Frenet system along the spherical curve $\gamma$ in the sense of covariant differentiation in the sphere. Namely,

$$
\begin{aligned}
\nabla_{T} T & =\sum_{i \neq j} u_{i} U_{i}, \\
\nabla_{T} U_{i} & =-u_{i} T, \quad i \neq j .
\end{aligned}
$$

More generally, if $u_{i_{1}}=c_{1}, u_{i_{2}}=c_{2}, \ldots, u_{i_{l}}=c_{l}$, then $\gamma$ lies on a $(p+1-l)$ dimensional sphere of radius $\left(c_{1}^{2}+c_{2}^{2} \ldots c_{l}^{2}\right)^{-1 / 2}$. In the exceptional case, $u_{i_{1}}=u_{i_{2}}=\ldots u_{i_{l}}=0, \gamma(s)$ lies on a $(p+1-l)$-plane. This corresponds to the case in classical Frenet theory in which the last $l$ curvatures vanish here the order of the curvatures matters.

As an application of the above discussion, we are now in a position to give a purely geometric version of our earlier variation formula, in the context of Euclidean and spherical curves.

Theorem 4. Variation of curvatures formula:

Let $M$ be Euclidean space of dimension $d=(p+1)$, or a round sphere of dimension $d=p$. Denote by $G$ the (constant) scalar curvature of $M$ (so $G=0$ or $G=\frac{1}{r^{2}}$ in the case of a sphere of radius $r$ ). Let $\gamma_{t}=X=$ $\alpha T+\sum_{i=1}^{d-1} x_{i} U_{i}$ describe a variation of a curve in $M$ through unit speed curves, where $U_{i}, i=1, \ldots,(d-1)$, is a natural frame along $\gamma(s, t)$. Then the induced variation of the associated natural curvatures $u_{i}$ is given by

$$
\begin{aligned}
\left(u_{i}\right)_{t}= & \left(\partial_{s}^{2}+G\right) x_{i}+\partial_{s}\left(\alpha u_{i}\right)+\sum_{l=1}^{d-1}\left(u_{l} \partial_{s}^{-1}\left(u_{l} \partial_{s} x_{i}-u_{i} \partial_{s} x_{l}\right)\right) \\
& +\sum_{j} c_{i, j} u_{j}, \quad i=1, \ldots,(d-1) .
\end{aligned}
$$

In the gauge term $\sum_{j} c_{i, j} u_{j}$, the $c_{i, j}$ are constants with $c_{i, j}=-c_{j, i}$.

Proof. Combine Theorem 1 with Propositions 3 and 6, after taking account of the above discussion.

We now merge the last few topics and consider the geometric evolution of curves in $R^{p+1}$ and $S^{p}$ corresponding to the above mKdV system. According to Theorem 3, the curve evolution $\gamma_{t}=X^{(2)}$ corresponds to the curvature evolution $Q_{t}=\tilde{X}^{(4)}$. In the present context, a fully geometric interpretation 
of this result is possible; note that the following theorem (like the previous one) is formulated entirely in terms of curve geometry - no Lie algebras!

Theorem 5. Motion of a curve $\gamma(s, t)$ in $R^{p+1}$ by the geometric evolution equation

$$
\gamma_{t}=-\left(\frac{3}{2} \kappa^{2} T+T_{s s}\right)=-\left(\frac{1}{2} k^{2} T+\sum_{i}\left(u_{i}\right)_{s} U_{i}\right),
$$

corresponds to curvature evolution by the $m K d V$ system

$$
\left(u_{i}\right)_{t}=-\left(\partial_{s}^{3} u_{i}+\frac{3}{2}|u|^{2} \partial_{s} u_{i}\right), \quad i=1, \ldots, p .
$$

Here, the functions $u_{i}$ are natural curvatures and $k^{2}=|u|^{2}=\sum_{i}\left(u_{i}\right)^{2}$ the squared curvature of $\gamma$. In particular, if one of the natural curvatures $u_{j}$ is initially constant along $\gamma$, then this condition is preserved, and $\gamma$ evolves on a sphere.

Proof. The first statement is easily obtained from the previous theorem, by direct computation. Alternatively, in view of Theorem 3, it suffices to note that in the present context,

$$
\begin{aligned}
X^{(2)} & =-\left\{-\frac{1}{2}[Q,[Q, A]]+\left[A, Q_{s}\right]\right\} \\
& =-\left\{-\frac{1}{2} \sum_{i, j} u_{i} u_{j}\left[X_{i},\left[X_{j}, A\right]\right]+\sum_{i}\left(u_{i}\right)_{s}\left[A, X_{i}\right]\right\} \\
& =-\left\{\frac{1}{2} \sum_{i}\left(u_{i}\right)^{2} A+\sum_{i}\left(u_{i}\right)_{s} Y_{i}\right\} \\
& =-\left(\frac{1}{2} \sum_{i}\left(u_{i}\right)^{2} T+\sum_{i}\left(u_{i}\right)_{s} U_{i}\right)=-\left(\frac{3}{2} \kappa^{2} T+T_{s s}\right) .
\end{aligned}
$$

It is also evident from the form of the coupled $\mathrm{mKdV}$ equations that the condition $u_{j}=$ constant is preserved in time, so the last statement follows.

Remark. In the case $k \neq 0$, the vectorfield $X^{(2)}$ is readily expressed in terms of the standard Frenet frame:

$$
X^{(2)}=-\left(\frac{3}{2} \kappa^{2} T+T_{s s}\right)=-\left(\frac{1}{2} \kappa^{2} T+\kappa_{s} N+\kappa \tau B\right) .
$$

Here, $\tau$ and $B$ are, respectively, the second curvature and second normal (in three dimensions, the torsion and binormal).

Expressed in this form, this vectorfield appeared in $[\mathbf{L}-\mathbf{P 1}]$ as the "next" term (above $X^{1}=k B$ ) in the localized induction hierarchy, and the connection to the (complex) $\mathrm{mKdV}$ equation was discussed. The planar version of 
this curve evolution and the connection to $\mathrm{mKdV}$ were considered in $[\mathbf{G}-\mathbf{P}]$ and in $[\mathbf{L}-\mathbf{P 3}]$. In $[\mathbf{L}-\mathbf{P} 4]$, the authors showed that the even terms of the $R^{3}$ localized induction hierarchy preserve curves lying on the sphere $S^{2}$, expressing these vectorfields in terms of the natural curvatures and frames. In [D-S], Doliwa and Santini show that $X=\frac{1}{2} \kappa^{2} T+\kappa_{s} N+\kappa \tau B$ describes an evolution of curves on $S^{3}$, and discuss corresponding curve evolution equations in spheres of arbitrary dimension. Our curve evolution equations (of this section) apparently coincide with those of [D-S] (though our approach is considerably different). On the other hand, the corresponding curvature evolutions of $[\mathbf{D}-\mathbf{S}]$ are increasing complicated as dimension of the sphere increases, and do not generally bear a close resemblance to the familiar (scalar) mKdV equation; we attribute this to their use of standard Frenet systems, rather than the natural Frenet systems we have employed here. Finally, our general formalism suggests a new perspective on the main conclusions of [D-S], regarding the characterization of integrable curve dynamics (as will be discussed in a future paper).

In this work we have described a Lie-theoretic construction of generalizations of the localized induction hierarchy and, in a special case, have shown how curvature evolution equations related to (but simpler in form than) those of Doliwa and Santini may be extracted as a subhierarchy. These geometric realizations of the Fordy-Kulish NLS systems have a structure remarkably similar to the $R^{3}$ LIE equations studied previously. Given the known relations between the localized induction hierarchy and classical geometric constructions, it is not unreasonable to expect that the geometric realizations of the Fordy-Kulish NLS systems will have similar interesting relations to geometry.

\section{References}

[Arn] V. Arnold, Mathematical Methods of Mechanics, Springer-Verlag, 1980.

[A-F] C. Athorne and A. Fordy, Generalized KdV and $m K d V$ equations associated with symmetric spaces, J. Phys. A, 20 (1987), 1377-1386.

[Bat] G.K. Batchelor, An Introduction to Fluid Dynamics, Cambridge University Press, 1967.

[Bis] R. Bishop, There is more than one way to frame a curve, American Mathematical Monthly, (March 1975), 246-251.

[C-I] A. Calini and T. Ivey, Bäcklund transformations and knots of constant torsion, J. Knot Theory and its Ramifications, 7(6) (1998), 719-746.

[D-S] A. Doliwa and P.M. Santini, An elementary geometric characterization of the integrable motions of a curve, Physics Letters A, 185 (1994), 373-384.

[F-K] A.P. Fordy and P. Kulish, Nonlinear Schrödinger equations and simple Lie algebras, Communications in Mathematical Physics, 89 (1983), 427-443. 
[G-L] O. Garay and J. Langer, Taimanov's motion of surfaces and Bäcklund transformations for curves, J. Conformal Geometry and Dynamics, 3 (1999), 37-49.

[G-G-K-M] C.S. Gardner, J.M. Greene, M.D. Kruskal and R.M. Miura, Method for solving the Korteweg-deVries equation, Physical Review Letters, 19 (1967), 10951097.

[G-P] R. Goldstein and D. Petrich, The Korteweg-de Vries hierarchy as dynamics of closed curves in the plane, Physical Review Letters, 67(23) (1991), 3203-3206.

[Has] H. Hasimoto, A soliton on a vortex filament, Journal of Fluid Mechanics, 51 (1972), 477-485.

[Hel] S. Helgason, Differential geometry, Lie groups, and symmetric spaces, Academic Press, New York, 1978.

[L] J. Langer, Recursion in curve geometry, New York J. Math., 5 (1999), 25-51.

[L-P1] J. Langer and R. Perline, Poisson geometry of the filament equation, J. Nonlinear Sci., 1 (1991), 71-93.

[L-P2] $\quad$ The filament equation, the Heisenberg model, and the non-linear Schrödinger equation, in W. Shadwick, P. Krishnaprasad, T. Ratiu, eds., Fields Institute Communications, Mechanics Day, AMS, Providence, RI, 1996, 181-188.

[L-P3] The planar filament equation, in W. Shadwick, P. Krishnaprasad, T. Ratiu, eds., Fields Institute Communications, Mechanics Days, AMS, Providence, RI, 1996, 171-180.

[L-P4] Local geometric invariants of integrable evolution equations, J. Math. Phys., 35 (1994), 1732-1737.

[Lan-S1] J. Langer and D. Singer, Knotted elastic curves in $R^{3}$, J. London Math. Soc., 30(2) (1984), 512-520.

[Lan-S2] _ Liouville integrability of geometric variational problems, Comm. Math. Helv., 69 (1994), 272-280.

[Lan-S3] L L Lagrangian aspects of the Kirchoff elastic rod, SIAM Review, 38 (1996), 605-618.

[M-W] J. Marsden and A. Weinstein, Coadjoint orbits, vortices, and Clebsch variables for incompressible fluids, Physica, 7D (1983), 305-323.

[Per1] R. Perline, Localized induction equation and pseudospherical surfaces, Journal of Physics A, 27 (1994), 5335-5344.

[Per2] L L L L L _ _ Lealized induction hierarchy and Weingarten systems, Physics Letters A, 220 (1996), 70-74.

[Pohl] K. Pohlmeyer, Integrable Hamiltonian systems and interactions through quadratic constraints, Comm. Math. Phys., 46 (1976), 207-221.

[Ric] R.L. Ricca, Rediscovery of the Da Rios equations, Nature, 352 (1991), 561562 .

[S-Y] N. Sasaki and Y. Yasui, Differential geometry of the vortex filament equation, J. Geom. Phys., 28(1-2) (1998), 195-207.

[S-Z] A. Shabat and V. Zakharov, Exact theory of two-dimensional self-focusing and one-dimensional self-modulation of waves in nonlinear media, Soviet Physics JETP, 34 (1972), 62-69. 
[Sym] A. Sym, Soliton surfaces and their applications, in 'Geometrical aspects of the Einstein equations and integrable systems', Lecture Notes in Physics, 239 (1985), 154-231.

[T-U1] C.L. Terng and K. Uhlenbeck, Poisson actions and scattering theory for integrable systems, preprint.

[T-U2] _ Schrödinger flows on Grassmanians, preprint math.dg/9901086.

Received September 2, 1998 and revised March 16, 1999.

Dept. of Mathematics

Case Western Reserve University

Cleveland OH 44106

E-mail address: jxl6@po.cwru.edu

Dept. of Mathematics and Comp. Sci.

DrEXEL UNIVERSITY

Philadelphia PA 19104

E-mail address: rperline@mcs.drexel.edu 